\title{
Monitoring of dispensed fluid with the quartz crystal microbalance (QCM) for the better control of inkjet or dispenser machine
}

\author{
Woosuck SHIN, Maiko NISHIBORI, Toshio ITOH, Noriya IZU and Ichiro MATSUBARA \\ National Institute of Advanced Industrial Science and Technology (AIST), Shimo-Shidami, Moriyama-ku, 463-8560 Nagoya
}

\begin{abstract}
Novel methods of materials deposition such as inkjet or dispenser can be used to integrate a variety of functional materials, light emitting materials, biomaterials, catalysts, while retaining their original functional activity. Coupled in with the high sensitivity of the quartz crystal microbalance (QCM), the average weight of one drop of deposited material can be monitored. The variations of the frequency changes of the QCM sensor are well correlated with the deposited mass of the droplet of the inkjet as well as dispenser. We are able to predict the dispensing volume with proper calibration of this QCM sensor.
\end{abstract}

(c) 2008 The Ceramic Society of J apan. All rights reserved.

Key-words: Inkjet, Dispenser, Direct writing, QCM sensor

[Received November 16, 2007; Accepted J a nuary 17, 2008]

Recent advancements on the nanoparticles of ceramics with various functional designs provide new prospects for direct printing or writing of 2 and/or 3 dimensional ceramic patterns and structures. ${ }^{1), 2)}$ These are much finer than the typical size of the current ceramic process, so we can call these ceramic microfabrication. Because the microfabrication of ceramic patterns by photolithographic techniques are time consuming and being an expensive process, there is a strong industrial need for direct digital printing. In other words, computer aided design, CAD, process, to simplify the processes and to reduce manufacturing costs, and the most well known processes are inkjet and dispensing techniques. ${ }^{3), 4)}$

Direct inkjet or dispensing, writing or printing, is a powerful non-contact, non-destructive rapid prototyping technique used for processing various materials. MEMS constructed devices have been reported to use these techniques and, some modern commercial process of plat panel display already take advantage of this technique. Direct writing of ceramic materials needs to be processed with ink materials that can convert to certain functional ceramics after solvent removal and heat-treatment. Two types of ink approaches have been pursued to attain the final forms, one is to use solution precursors and the other is to use suspensions of ceramic powders, the ceramic pastes or slurry. While most inkjet printings use the solution-type inks, the dispensing techniques are more adoptable for the use of ceramic suspensions.

Regardless of the ink types, the piezoelectric inkjet printing reproducibly formed from sub to $10 \mathrm{pL}$ drops for a typical deposition process. This drop size seems popular considering the higher reproducibility for small-scale pattern fabrication. For the dispensing process, the dispensing speed rather than the drop size of the ink is a more important parameter and it typically varies from 0.01 to $1 \mathrm{~nL} / \mathrm{s}$, mainly depending on the nozzle diameter. In most cases, to control the dispensing volume is the most critical issue for fine pattern fabrication and also for product level fabrication with controlled quality level.

Though the popular method of monitoring the inkjetprinted volume is to measure millions of drops by micro- balance, it is a very time-consuming process and the condition could change after the long dispensing. Imagine the 1 microgram drop, which can be measurable by microbalance, will be obtained by injections of 1 million of $\mathrm{pL}$ drops. If the droplets were injected at a frequency of $200 \mathrm{~Hz}$, then one shell wait for $5000 \mathrm{sec}$ or 80 minutes to monitor the drop volume. In this report, dispensing of droplets coupled in conjunction with the weight sensing by a quartz crystal microbalance (QCM) has been studied to test the fast and novel method of in-situ monitoring of dispensing volume for the direct writing technique.

Figure 1 shows the QCM sensor system, THQ 10D, Tamadevice Inc., used in this study. The QCM sensor element was an AT cut of quartz, where the resonance frequency $f_{\mathrm{o}}$ is proportional to its thickness, $t$, and the $t=0.185 \mathrm{~mm}$, $f_{\mathrm{o}}=9 \mathrm{MHz}$, was used. The working principle of the sensor is that the attached drops on the sensor surface increased its mass, by the change of $\Delta m$, and this change of mass is a linear function of the resonance frequency changes, $\Delta f$, as

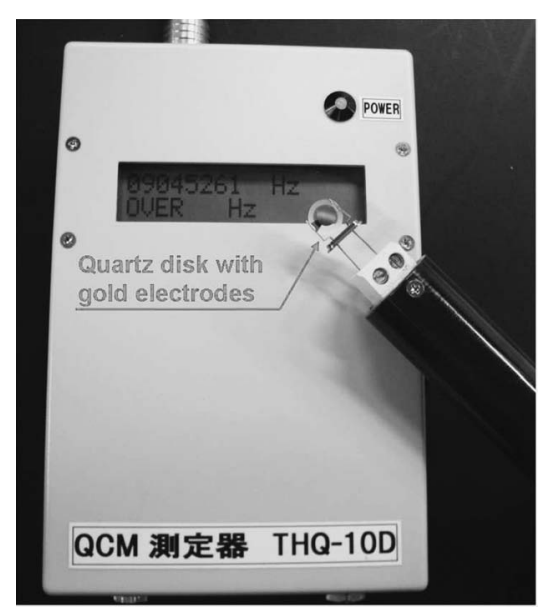

Fig. 1. Snap of QCM system which is commercially available. The drop is deposited on the small $1 \mathrm{~cm}$ disk of quartz with gold electrodes, and the frequency counter (left box) displays the $\Delta f$. 


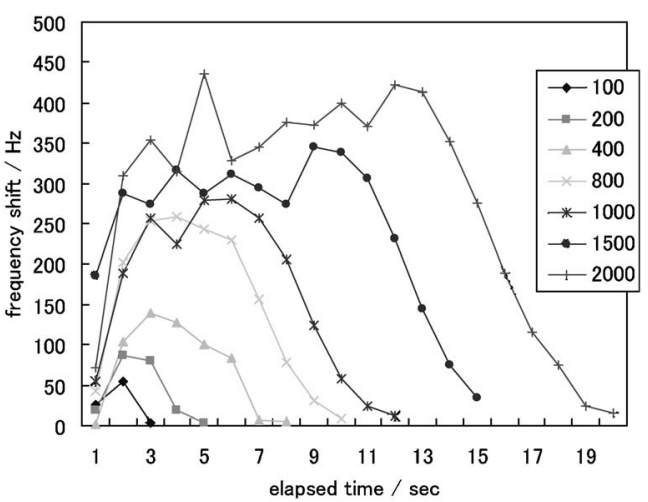

Fig. 2. Change of the frequency shift of the QCM sensor after 100 to 2000 pulse drops injected.

shown in equation 1 , so called the Sauerbrey equation,

$$
\Delta f=\frac{-2 \times f_{\mathrm{o}}^{2}}{A \sqrt{\mu \times \rho}} \Delta m
$$

where the $A$ is the electrode area, $\mu$ and $\rho$ are shear stress and density of quartz, $2.947 \times 10^{10} \mathrm{~kg} \cdot \mathrm{m}, 2648 \mathrm{~kg} / \mathrm{m}^{3}$, respectively. With the electrode data, the simple equation of $\Delta f(\mathrm{~Hz})=1153 \Delta m(\mathrm{ng})$ can be used in this study.

We have tested this sensor for both inkjet and dispenser systems, at $29^{\circ} \mathrm{C}$ and around $40 \%$ relative humidity. The inkjet setup consisted of a piezoelectric ink-jet nozzle manufactured from Microjet Inc., and the solution used for this inkjet is $i$-propanol. The QCM sensor has been set below the nozzle with the gap between the nozzle and the sensor surfaces maintained at $0.5 \mathrm{~mm}$ and uniform ejection of the droplets was performed by applying voltage impulse for the period of $0.618 \mathrm{~ms}$ with delay of $0.605 \mathrm{~ms}$.

The sensor response was delayed for seconds as shown in Fig. 2. Hundreds of inkjet droplets of the solution have been injected in a second, but the response of the sensor delayed several seconds and reached its highest level at around from 2 to $7 \mathrm{~s}$. As time passed, the level decreased again and disappeared after. This seems due to the evaporation of the solution of $i$-propanol.

Although we have uncertainty of the highest value of this signal, these values of $\Delta f$ are considered to be saturated ones and plotted for various drop pulse counts, as shown in Fig. 3. Using the simple equation of $\Delta f(\mathrm{~Hz})=1153 \Delta \mathrm{m}$ (ng), the change of the mass, $\Delta m$, are plotted by right-side $y$ axis. From this result, the single droplet can be calculated to be $0.25 \mathrm{ng}$. Looking closer into the lines, the inclination of the plot can be found to change between the pulse of 800 . This could suggest to us that the correlation is not linear and changes if the mass of droplets increase, and became rather insensitive.

This seems much more prominent if we use large volumes of droplets. The dispenser droplet is a typical example. Water and ceramic paste are dispensed by an air-pressure dispenser 808FX MUSASHI Engineering Inc., with a needle inner diameter of $0.1 \mathrm{~mm}$. The pressure used for ceramic paste was $50 \mathrm{kPa}$, but the negative pressure was applied for water dispensing. For the characterization of deposited droplet volume, a contact angle measurement system, DropMaster-500, Kyowa Interface Science Co., Ltd, was used. The dispensed droplet volume of the water and ceramic paste

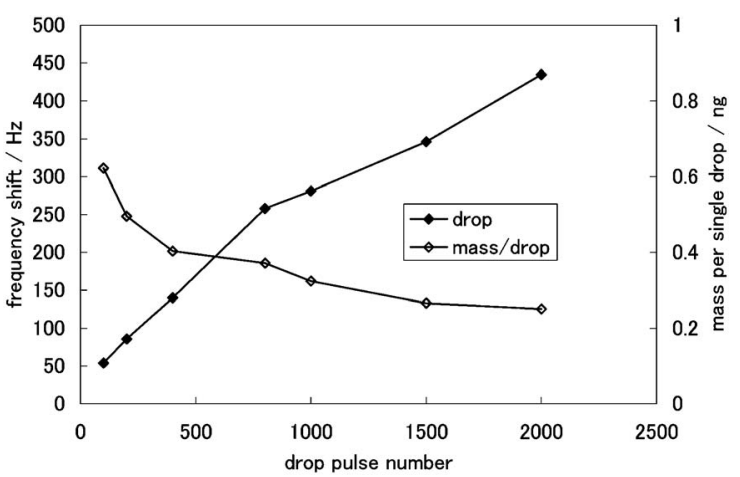

Fig. 3. In-situ quantitative monitoring of the inkjet droplets using QCM sensor device, showing the frequency shift of QCM was plotted with the number of drop pulses.

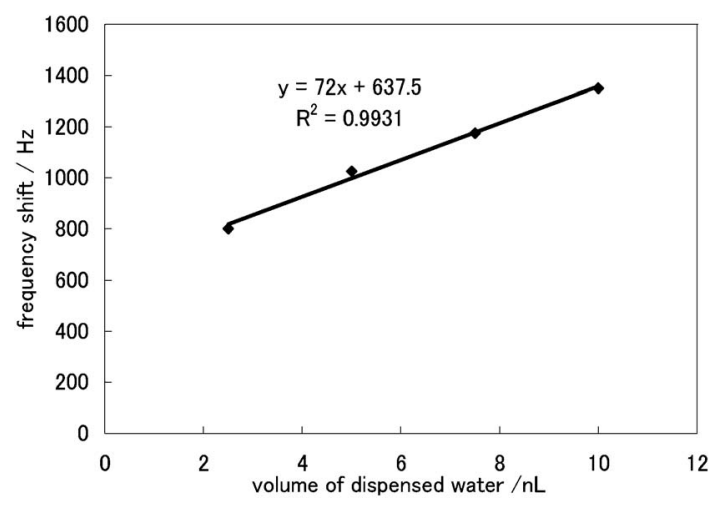

Fig. 4. Frequency shift of QCM with the dispensed volume of water.

are calculated from the data of the contact angle between the droplet and the substrate, and the shape of the drop.

Figure 4 shows the frequency shift of QCM with the volume of deposited water. In this case we could not use the previous simple equation, of $\Delta f(\mathrm{~Hz})=1.153 \Delta m(\mathrm{ng})$, but the correction that $\Delta f(\mathrm{~Hz})=0.072 \Delta m(\mathrm{ng})$ was fitted. This big deviation of 16 times is unexpected but it is still linear in this narrow range. From the above results, we could suggest that the QCM sensor system can be used after the proper calibration process.

Furthermore, if the dispensed liquid has a higher viscosity, the situation seems to more serious. Figure 5 shows the frequency shift of QCM with the volume of deposited ceramic paste. The ceramic paste used in this study is the catalyst of $\mathrm{Pt} / \mathrm{Al}_{2} \mathrm{O}_{3}$ mixed up with terpineol and ethyl cellulose, applied for micro devices as reported previously. The preparation and rheological characteristics of the paste have been reported in detail ${ }^{5)}$ and typically the viscosity is over $1 \mathrm{~Pa} \cdot \mathrm{s}, 1000$ times higher than that of water.

As we evaluated the paste volume from the analysis of the deposited drop, with the dispensing condition of $50 \mathrm{kPa}, 2 \mathrm{~s}$, the ceramic paste volume deposited was $40 \mathrm{~nL}$. The density of this ceramic paste is $2.0 \mathrm{~g} / \mathrm{cc}$ and then the deposited mass was 80 micrograms. In this case, the relationship between the frequency shift and mass change changes further. This paste may deposit on the sensor as a shape of the droplets not 


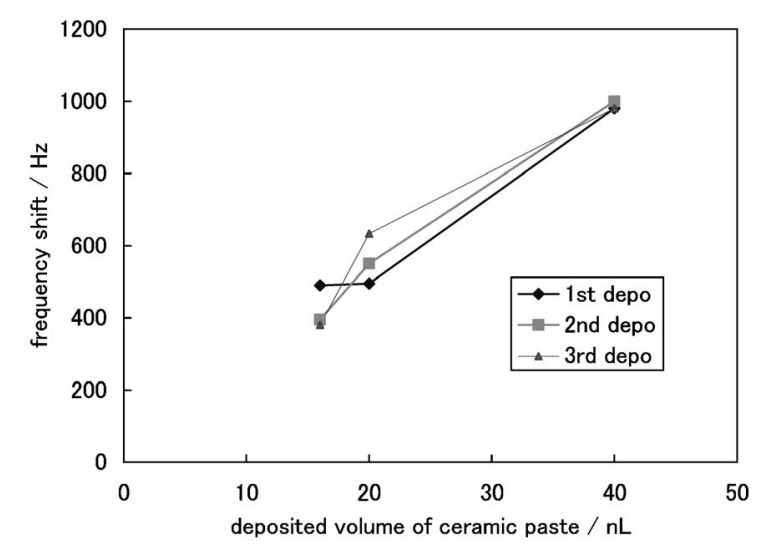

Fig. 5. Frequency shift of QCM with the dispensed volume ceramic paste. By QCM data, it can be checked that three dispensing test resulted similar drops reproducibly.

spreading on the surface and may change this relationship further. The relationship of the frequency and mass differ approximately 80 times from the calculated one, $\Delta f(\mathrm{~Hz})=$ $0.0125 \Delta m(\mathrm{ng}))$. Similar to the case of water, we could use this QCM system with the proper calibration although fittings are necessary for different droplet materials. Furthermore, we have confirmed that three different dispensing times with the same condition, $50 \mathrm{kPa}$ and $2 \mathrm{~s}$, have per- formed almost same deposition volume of drops, as shown in Fig. 5. This drop monitoring also can be used for the microfabrication quality control of ceramic paste processing.

We have demonstrated an in-situ monitoring of the droplet mass of inkjet or dispenser drops on the QCM sensor. The variations of the frequency changes of the QCM sensor are well correlated with the deposited mass of the droplet of the inkjet as well as the dispenser. We are able to predict the dispensing volume with proper calibration. Further studies of the contact angle and wetting characteristics of the drops on the sensor electrode surface are necessary for the better precision monitoring, and we hope the feedback system controlling the dispensing condition such as the driving voltage or pressure with this novel QCM system is expected to be promoted by this report of ours.

\section{References}

1) Y. Masuda, J. Ceram. Soc. Japan, 115, 101-109 (2007).

2) M. Heule, S. Vuillemin and L. Gauckler, Adv. Mater., 15, 1237-1245 (2003).

3) H. Yokoyama, N. Katoh, T. Hotta, M. Naito and S. Hirano, $J$. Ceram. Soc. Japan, 111, 262-266 (2003).

4) J. A. Lewis and G. M. Gratson, Mater. Today, 7, 32 (2004).

5) W. Shin, M. Nishibori, K. Tajima, L. F. Houlet, Y. Choi, N. Izu, N. Murayama and I. Matsubara, Sens. Actuators, B, 118, 283-291 (2006). 